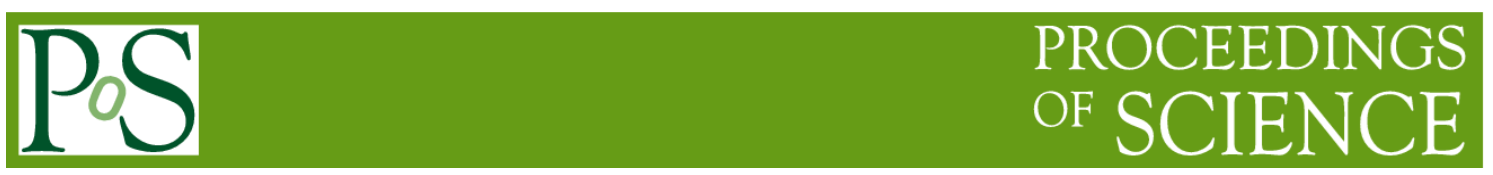

\title{
Geant4 simulation of sFLASH experiment
}

\author{
Jian-Jung Huang ${ }^{1 \mathrm{a}}$, for sFLASH collaboration \\ ${ }^{a}$ LeCosPA, National Taiwan University \\ No. 1 Sec. 4, Roosevelt Rd., Taipei 10617, Taiwan \\ E-mail: njinee@hotmail.com
}

\begin{abstract}
The goal of Super Fluorescence Air SHower (sFLASH) is to reduce the current fluorescence yield systematic uncertainty to improve energy measurement of ultra-high energy cosmic rays. The experiment was performed at SLAC National Accelerator Laboratory to measure the air fluorescence yield from extensive air showers. A set of alumina bricks is placed in front of the beam pipe as the target to generate a cascade in the bricks. When the charged particles of cascade escape from the target, they excite the air molecules and emit the fluorescence. Several photon multipliers collect the fluorescence in the dark room to estimate the fluorescence yield. In this simulation, the proper geometry of this experiment is built with Geant4, which is the simulation toolkit for high energy particle interactions.
\end{abstract}

Bexco, Busan, Kore

\section{${ }^{1}$ Speaker}




\section{Introduction}

\section{Ultra-high energy cosmic rays}

The history of cosmic ray observation started from 100 years ago [1-3]. Due to the extremely small flux of the ultra high energy comic ray (UHECR), some properties of UHECR are still unclear. More precisely measurement are required to explore the UHECR physic. Building a large scale observation, such as Pierre Auger Observtory [4], and Telescope Array Observtory [5-7], is the most direct stratagy to understand the UHECR with the low flux. Systematic uncertainties of energy scale reconstruction include several parts: 1) fluorescence yield, 2) detector calibration, 3) atmospheric transmission and so on. The precise measurement of the energy of UEHCR can help us to figure out the acceleration mechanism or the origins of these extremely energetic particles.

\section{sFLASH experiment}

The air florescence yield is one of the key factors that determined the uncertainty of energy of UEHCR. The primary goal of the sFLASH experiment is to reduce the systematic uncertainty on the air fluorescence yield. Six photomultiplier tubes (PMTs) are placed far away from the electron beam and surrounded by the lead bricks. SLAC national accelerator laboratory (SLAC) provides

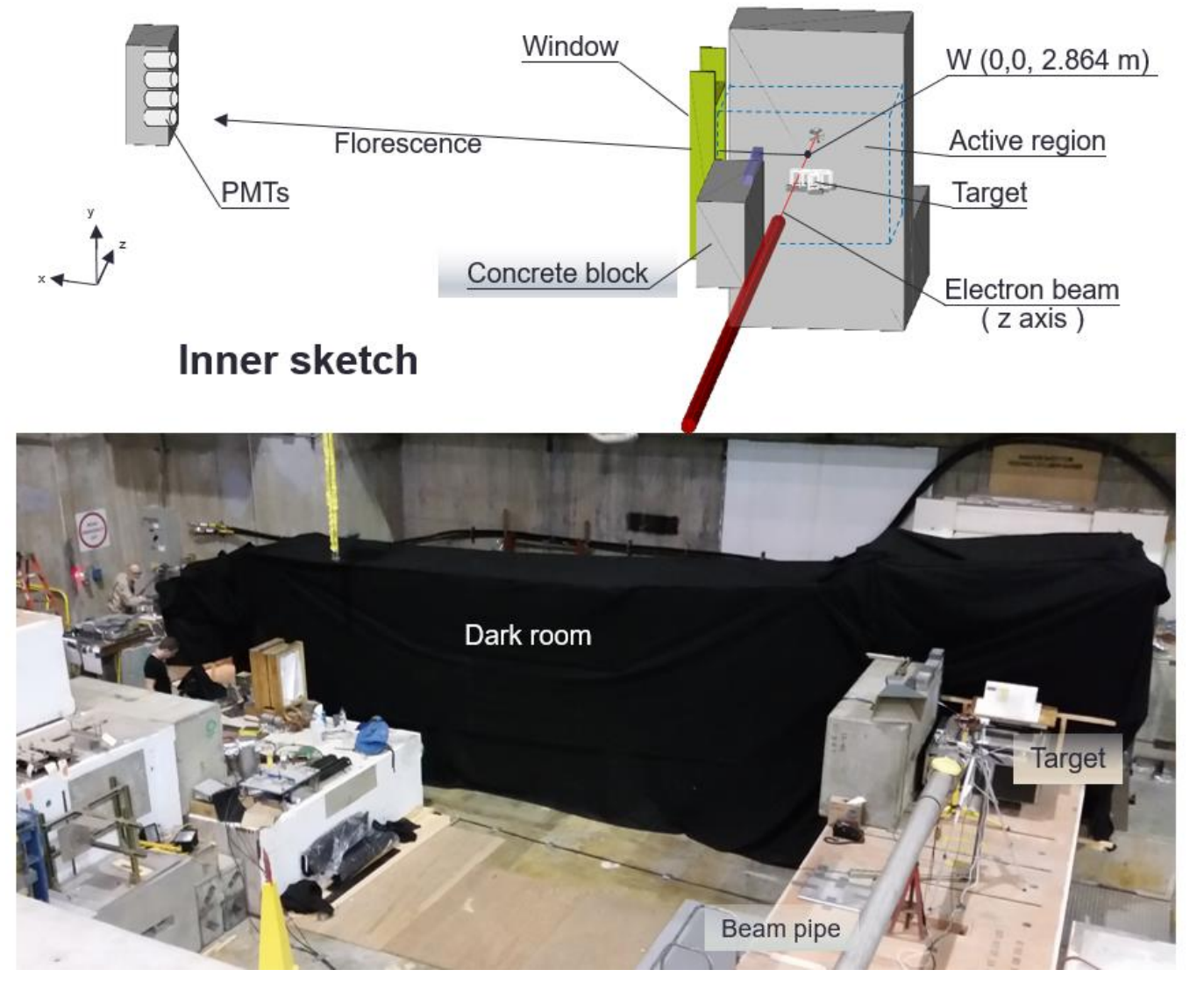

Figure 1 sFLASH experiment in SLAC \& inner sketch

the adjustable electron beam as particle source and each bunch of electron beams contains $10^{9}$ electrons. The electron beam comes out from the vacuum pipe and enters the target, $\mathrm{Al}_{2} \mathrm{O}_{3}$ bricks, to create cascades. The sFLASH target thickness can be changed by rearranging the alumina bricks; the different thickness of the target implies a different slice of the shower age. On the other words, by arranging the thickness of target, sFlash can extract the shower information of a specify shower age. When energetic particles escape from target and propagate in the air; the particles 
excite the atoms and emit the isotropic fluorescence photons in active region. Six PMTs located 10 meters away transverse to the beam axis to collect the fluorescence photons within active region (Figure 1). Two concrete blocks are placed in fornt of PMTs to veto the large anlge scattering of eneergtic particles either from target or the rest area. PMTs only accept the photons through a window on dark room from active region. In our simulation, five different energies of electrons are considered as the beam source. The energetic electrons injected from the beam pipe stickle the $\mathrm{Al}_{2} \mathrm{O}_{3}$ targets. The concrete floor and wall behind the beam dump are also included in the simulation to include the effect of the large angle backward scattering of particles. In the simulation, primary electrons come one by one into the target and generate cascades which deposite its energy into enviroment. The program records total energy deposited in the target, air, and the beam dump. The coordinate system is Cartesian. The electron beam goes along z-axis and $\mathrm{x}$-axis direction is defined by towarding to PMT from beam line.

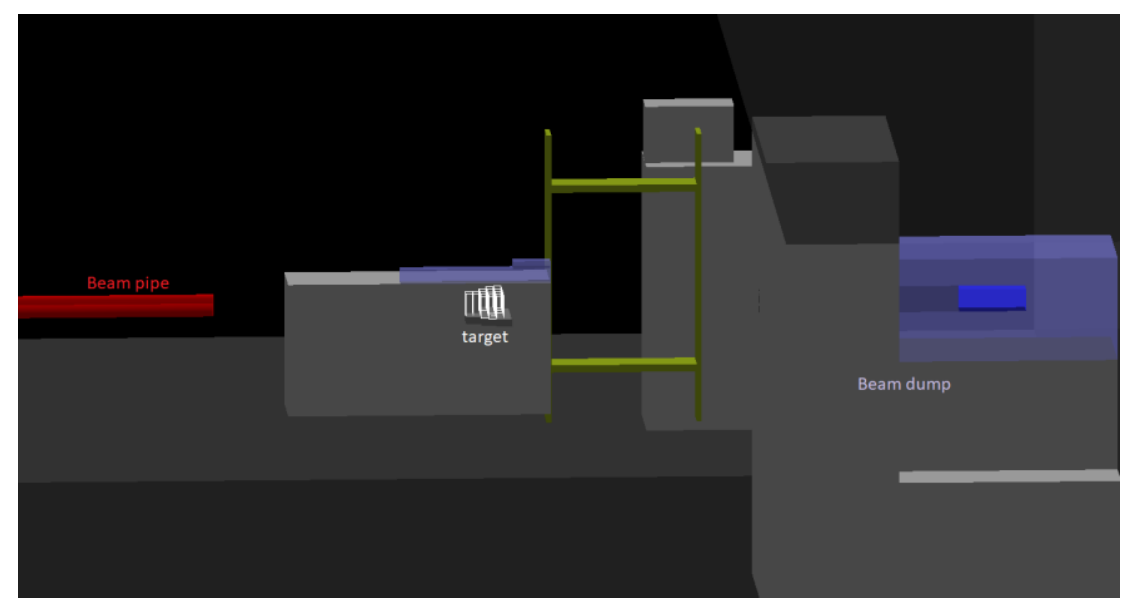

Figure 2: Geant4 model of sFLASH experiment

\section{$\mathrm{Al}_{2} \mathrm{O}_{3}$ target}

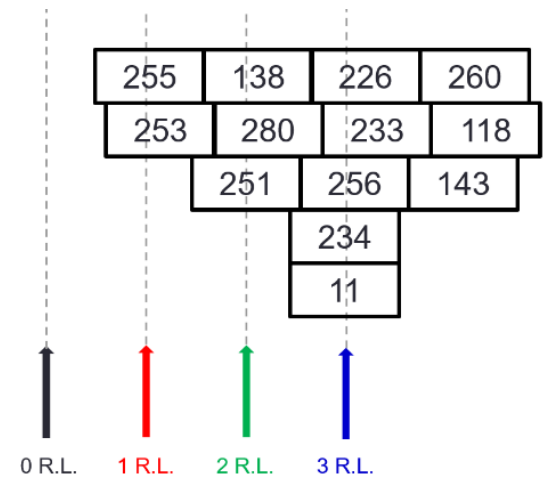

Figure 3 Configuration of $\mathrm{Al}_{2} \mathrm{O}_{3}$ bricks

The $\mathrm{Al}_{2} \mathrm{O}_{3}$ bricks have different densities and their arrangement is shown in Figure 3. In this configuration it has 3 different thickness of target that is equivalent to $1.29,1.95$, and 3.25 radiation length (R.L.). In the later discussion, they are labeled as 1, 2, and 3 R.L. And 0 R.L. simply means a missed shot. 


\section{Energy deposition \& florescence photon yield}

After an electron beam coming out from the vacuum pipe, primary electrons would first travel through air and later through the $\mathrm{Al}_{2} \mathrm{O}_{3}$ target generating the secondary cascade shower. These bunch energetic particles excites air molecules and the excitation result emission of florescence photons. Assuming the total deposition of energy in a given volume is $E(x)$ and total number of photons collected by a PMT 10 meter away from electron beam line is $N_{\text {photon }}$. It follows the equation that

$$
N_{\text {photon }}=\int d^{3} x \eta_{\text {air }} \frac{d E(x)}{d V} \frac{\Delta \Omega(x)}{4 \pi},
$$

where $\eta_{\text {air }}$ is the florescence photon yield and $\Delta \Omega(x)$ is the corresponding solid angle at a position $\mathrm{x}$. This solid angle can be aproximated by $\frac{A_{P M T} \cos \theta}{r^{2}}$ if it is small enough or the PMT is suficiently far away comparing to PMT aperture with $r=\left|\boldsymbol{x}-\boldsymbol{x}_{P M T}\right|, \cos \theta=\boldsymbol{x} \cdot \frac{\boldsymbol{n}}{\left|\boldsymbol{x}-\boldsymbol{x}_{P M T}\right|}$, and $\boldsymbol{n}$ is a normal vector of a PMT.

Then, equation becomes

$$
N_{\text {photon }}=\eta_{\text {air }} \int d^{3} x \frac{d E(x)}{d V} \frac{A_{P M T} \cos \theta}{4 \pi r^{2}},
$$

Defining an integration $I$ by

We have photon florescence photon yield by

$$
I=\int d^{3} x \frac{d E(x)}{d V} \frac{\cos \theta}{4 \pi r^{2}}
$$

$$
\eta_{\text {air }}=\frac{N_{\text {photon }}}{A_{P M T} I} \text {. }
$$
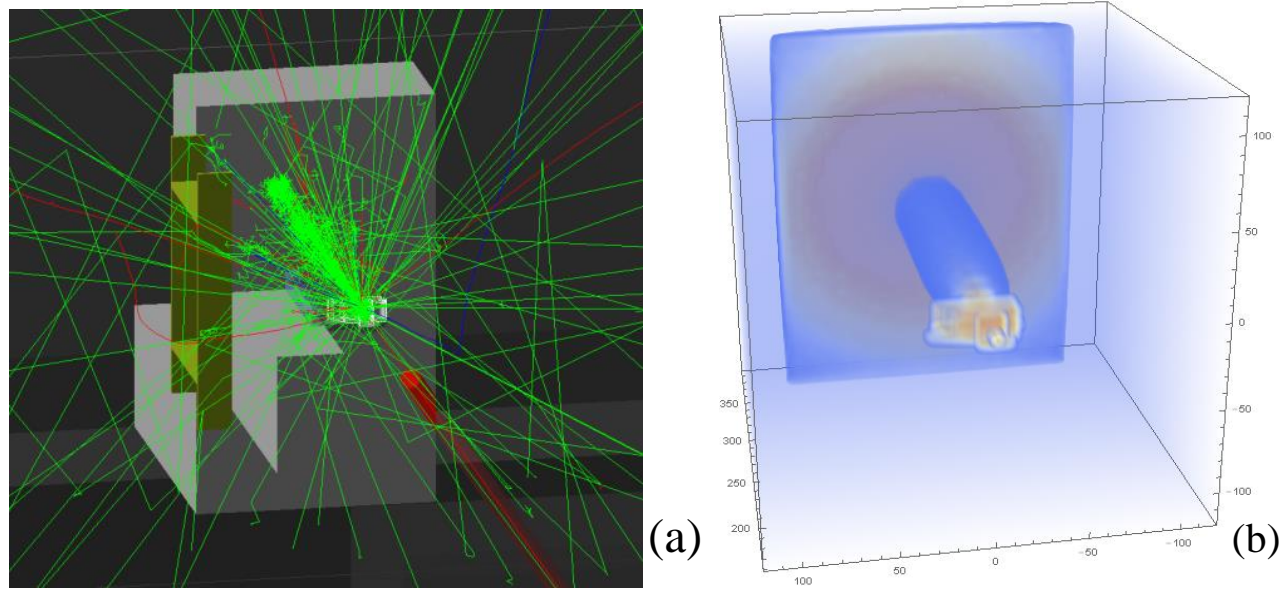

Figure 4 Geant4 simulation \& energy deposition in $3 D$ density plot 


\section{Result \& discussion}

\section{Energy deposition}

In Geant 4 framework, every particle and its daughter particle can be tracked. It is visaulized as shown in Figure 4a. Most of the track in the simulation are gamma particles' represented in green lines. The rests are red line for negtive charged particles and blue line for positive charged particle.

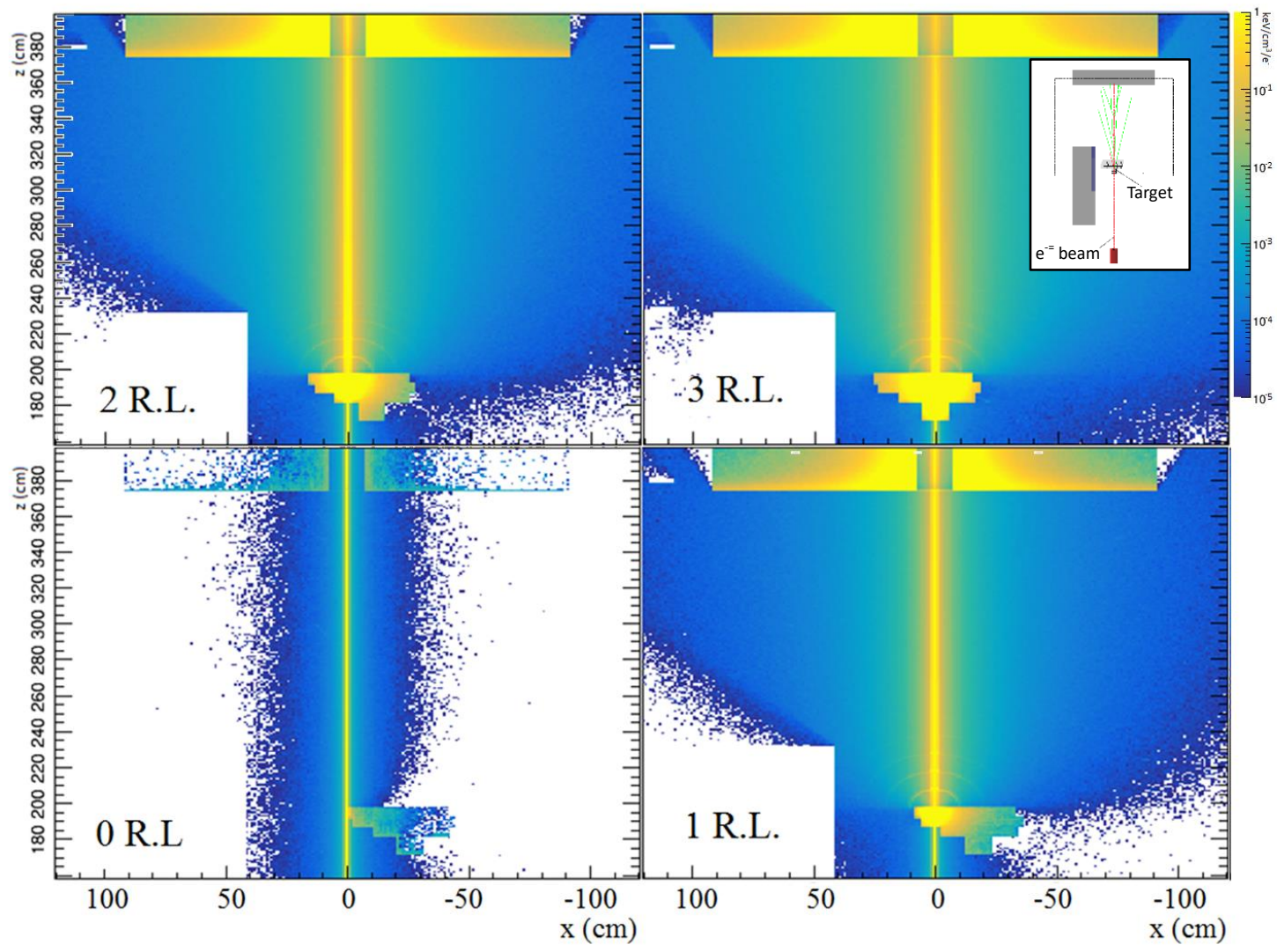

Figure 5 The result of electrons hitting different thickness of $\mathrm{Al}_{2} \mathrm{O}_{3}$ bricks. The distribution of total energy deposition spreads with an increasing of target length and the concrete block absorbs excessive particles.

The deposited energy are recorded by 2 different 3-dimensional histograms. One records detailed structure of the energy distribution with a $1 \mathrm{~cm}^{3}$ unit volume but only covers volume around the active region, the target, and the beam dump. The other records the distribution over much bigger space but at lower resolution with a $1000 \mathrm{~cm}^{3}$ unit volume. The deposited energy distribution of simulation of primary electron with $10.49 \mathrm{GeV}$ is represented in Figure $3 \mathrm{~b}$ as a 3D density plot. In the Figure 5, it shows the result of electrons hitting different thickness of $\mathrm{Al}_{2} \mathrm{O}_{3}$. One can easily tell that the spreading and the total energy deposition go up with an increasing of target length and the concrete block works well for absorbing excessive particles.

We investigated the energy distribution for different targets and also compared it with and without shielding concrete blocks. We took point W (see figure 1) as the origin and the direction of propagation towards the PMTs. It shows that shielding lowers the energy deposition by a factor of 5 , see figure 6 . 


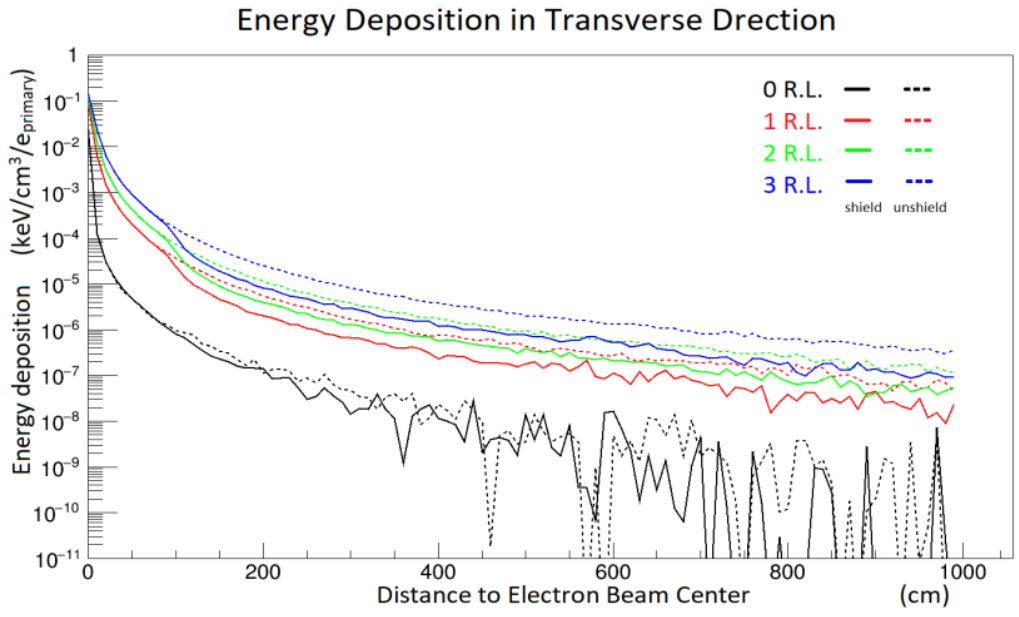

Figure 6 Energy deposition along in the transverse direction

\section{Florescence photon yield}

In this part, we discuss the value of integral $I$ at different energies of the primary electron and

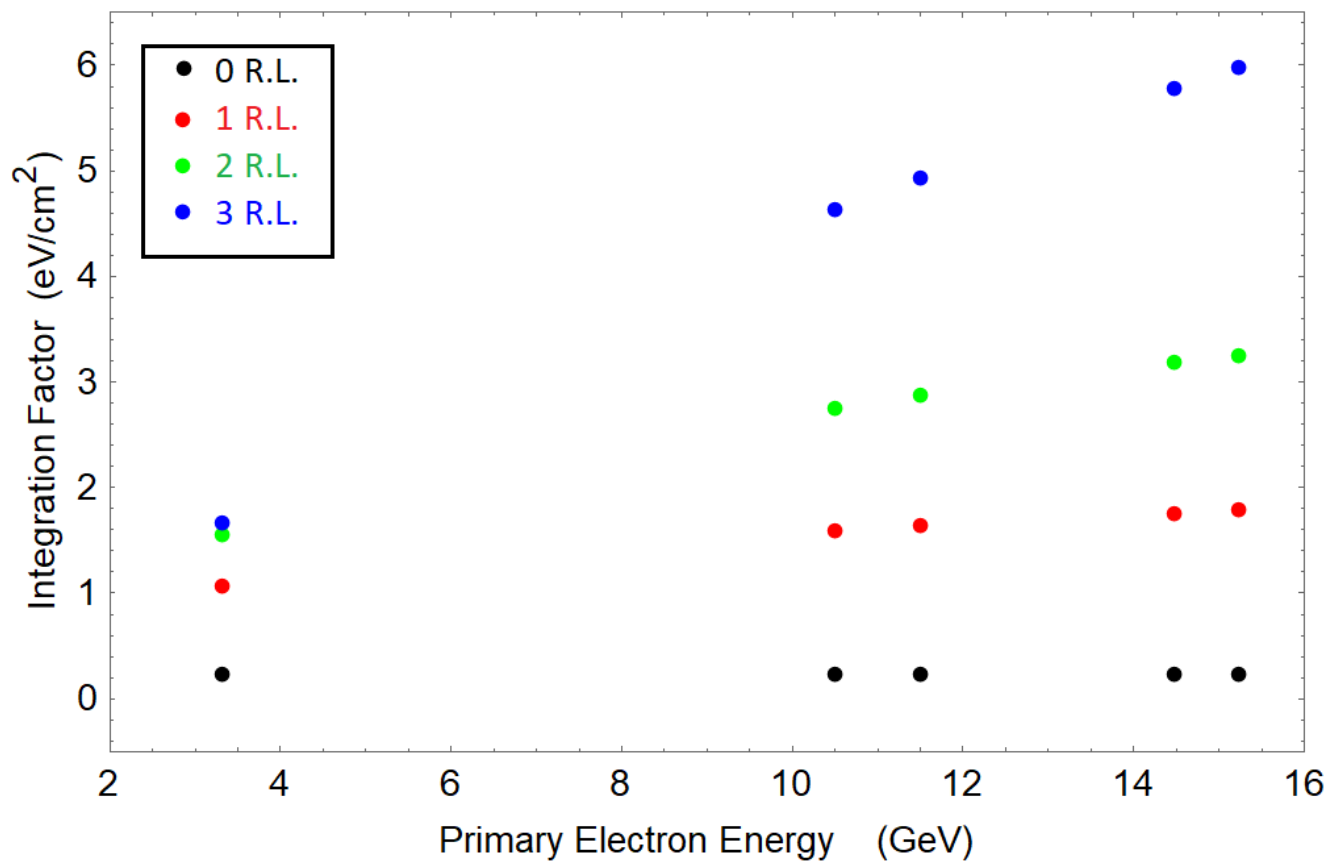

Figure 6 Integration factor with differnet primaty e energy \& thickness of $\mathrm{Al}_{2} \mathrm{O}_{3}$. Energy of electron beam is $3.15 \mathrm{GeV}, 10.49 \mathrm{GeV}, 11.49 \mathrm{GeV}, 14.48 \mathrm{GeV}$, or $15.23 \mathrm{GeV}$. We can extract florescence photon yield $\eta_{\text {air }}$ by effective area of PMT and the number of collected photon by PMT in each run.

different target thickness. The result is shown in Figure 7. The fluorescence yield can be obtained by multiplying the integral I by the PMT effective area and normalizing by the number of photons according to the equation shown above 


\section{Reference}

[1] B.R. Dawson, M. Fukushima, and P. Sokolsky, Past, Present and Future of UHECR Observations, (B.R. Dawson)

[2] J. F. Ormes, editor, Centenary Symposium 2012: Discovery of Cosmic Rays , volume 1516 of American

[3] K.-H. Kampert and A. A. Watson, Eur. Phys. J. H, 37, $359\{412$ (2012), arXiv:1207.4827.

[4] M. Nagano, T. Hara, Y. Hatano, et al., J. Phys. G: Nucl. Phys., 10, 1295\{1310 (1984).

[5] H. E. Bergeson, G. L. Cassiday, T.-W. Chiu, et al., Phys. Rev. Lett., 39, 847\{849 (1977).

[6] R. M. Baltrusaitis, R. Cady, G. L. Cassiday, et al., Nucl. Instrum. Methods Phys. Res., Sect. A, 240, 410\{428 (1985).

[7] G. Thomson, Nucl. Phys. B Proc. Suppl., 136, $28\{33$ (2004).

[8] J. Abraham, M. Aglietta, I. C. Aguirre, et al., Nucl. Instrum. Methods Phys. Res., Sect. A, 523, 50-95 (2004).

[9] P. Sokolsky and G. B. Thomson, J. Phys. G: Nucl. Phys., 34, R401 \{R429 (2007), arXiv:0706.1248.

[10] S.Agostinelli et al. Nuclear Instruments and Methods in Physics Research A 506 (2003) 250-303

[11] J. Allison et al. IEEE Transactions on Nuclear Science 53 No. 1 (2006) 270-278

[12] J.Allison et al. Nuclear Instruments and Methods in Physics Research A 835 (2016) 186-225 